\title{
Correction to: In the eye of the beholder: how self-other agreements influence leadership training outcomes as perceived by leaders and their followers
}

\author{
Karina Nielsen $^{1}$ • Susanne Tafvelin ${ }^{2}$ - Ulrica von Thiele Schwarz ${ }^{3,4}$ - Henna Hasson ${ }^{3}$ \\ Accepted: 1 February 2021 / Published online: 8 February 2021 \\ (C) Springer Science+Business Media, LLC, part of Springer Nature 2021
}

\section{Correction to: J Bus Psychol.}

https://doi.org/10.1007/s10869-020-09730-3

In the paper In the Eye of the Beholder: How Self-Other Agreements Influence Leadership Training Outcomes as Perceived by Leaders and Their Followers, DOI: https://doi. org/10.1007/s10869-020-09730-3, Professor von Thiele Schwarz's surname was misspelled Schwartz. The correct spelling is Schwarz.

The full name reads:

Given name: Ulrica.

Last name: von Thiele Schwarz.

The original article has been corrected.

Publisher's Note Springer Nature remains neutral with regard to jurisdictional claims in published maps and institutional affiliations.

Karina Nielsen

k.m.nielsen@sheffield.ac.uk

Susanne Tafvelin

susanne.tafvelin@umu.se

Ulrica von Thiele Schwarz

ulrica.schwarz@mdh.se

Henna Hasson

henna.hasson@ki.se
IWP, Sheffield University Management School, University of Sheffield, Sheffield S10 1FL, UK

2 Umeå University, 90187 Umeå, Sweden

3 Department of Learning, Informatics, Management and Ethics, Karolinska Institutet, Tomtebodavägen 18a, 171 77 Stockholm, Sweden

4 School of Health, Care and Social Welfare, Mälardalen University, HÖGSKOLEPLAN 1, 72123 Västerås, Sweden 